\title{
The Effects of a Small Local Change in Phase Velocity on the Propagation of a VLF Radio Signal
}

\author{
D. D. Crombie
}

Contribution From the Central Radio Propagation Laboratory, National Bureau of Standards, Boulder, Colo.

(Received December 6, 1963)

\begin{abstract}
Changes in the propagation of a VLF signal due to the changes in phase velocity caused by a small localized depression in the height of the ionosphere along or near the line joining the transmitter and receiver are investigated using elementary diffraction theory. It is shown that both the phase and amplitude of the received signal may be changed if the horizontal extent of the depression is great enough and if it is sufficiently close to the path. If the depression is at greater distances from the path, the amplitude and phase of the received signal merely oscillates about the undisturbed values. An account is given of the use of Cornu's spiral in estimating the effects of a particular disturbance.
\end{abstract}

\section{Introduction}

It is sometimes of interest to be able to estimate how the phase and amplitude of a VLF radio signal will be altered if the height of a small area of the ionosphere, along or near the path of the radio wave, is altered. Such ionospheric height changes, in the form of depressions, might be caused, for example, by the localized "dumping" of electrons from the radiation belts or by a small nuclear explosion at heights such that the resulting prompt gamma and $x$ radiation produce ionization at limited ranges.

Now, in practice, the signal from a distant transmitter is comprised of energy received over a finite range of azimuth angles and not merely that received along the line between receiver and transmitter. Thus, a disturbance in the ionosphere must subtend an angle at the receiver which is comparable with the azimuthal sector in which the signal is received in order to produce an appreciable effect on the received signal. Otherwise energy will be diffracted around the disturbance, and it will cause little effect.

Again, the width of the wavefront which makes the significant contribution to the received signal depends on its distance from the receiver and transmitter and is a maximum when it is equidistant from them. Thus, if the ionospheric disturbance is sufficiently small and of fixed dimensions, the effects it produces will depend on its location along the path. It will be seen, therefore, that it is not permissible, in general, to apply an earlier result obtained by Wait [1961] for an infinitely wide disturbance to a disturbance which is of limited width (perpendicular to the path).

The situation is further complicated, since a disturbance of the type outlined above, occurring at random, is more likely to occur on one side or the other of the path rather than on it. Nevertheless, in such a case, the propagation of the signal is still likely to be affected, although only slightly if the disturbance is sufficiently remote from the path.
In this paper, these effects will be investigated by using an approximate method based on Fresnel diffraction theory. In order to clarify the work, several simplifying assumptions will be made. First, it will be supposed that propagation is two dimensional (in the horizontal plane), and that the earth is flat. It will also be assumed that all distances are sufficiently large that only the first order waveguide mode need be considered. The effect of a local change in height of the ionosphere may then be associated with a local change in phase velocity of the single propagating mode [Wait, 1961]. 'This assumption is reasonable if the slope in the ionosphere is small. In any case, it will be assumed that the depression does not generate significant higher order modes and that refraction can be ignored. Finally, it will be assumed that even though the ionospheric disturbance is of finite size, its effects can be represented by a "lumped" phase shift on that portion of the wavefront which passes through the center of the disturbance and which is bounded in the transverse direction by the edges of the disturbance.

The value of the phase shift introduced by a particular change in the ionosphere depends of course on the change in phase velocity which is produced. This then depends on the size and shape of the ionospheric disturbance. In general there is an infinite combination of sizes and shapes of disturbances which could produce the same lumped phase shift. The special case of a disturbance in which the height of reflection of VLF radio waves cosinusoidally with distance has been treated by Wait [1961]. Thus, in this paper it will be assumed that the actual phase change $\varphi$ suffered by a ray of infinitesimal width in passing under a particular ionospheric depression has already been determined, by using methods such as that discussed by Wait [1961]. This paper will be solely concerned with investigating the effects of changes in the width and the position of the disturbance. In particular the effects of a disturbance on the phase and amplitude of the received signal 
will be calculated for two specific cases: (1) when the center of a disturbance of varying width is on the great circle path, and (2) when a disturbance of fixed width moves across the path. It will also be shown that the effects of a small circular disturbance tend to be proportional to the square of its diameter. Thus small disturbances are much less likely to produce observable effects than an inappropriate or naive application of Wait's [1961] calculations would indicate. Finally, a consequence of including the effect of the earth's curvature will be discussed.

A related problem has been discussed by Wait [1961]. He was primarily concerned with the effects of a disturbance which, while of finite dimensions along the direction of propagation, was infinitely large in the transverse direction. Wait was primarily concerned with the change in phase delay which would be expected as the longitudinal length and depth of the depression were varied. He found, inter alia, that the phase change produced by a depression of this form was independent of its position between the transmitter and receiver. Recently, Wait [1964 a, b] has extended these results to cover the case of a disturbance of finite width as it moves across the path, and he has presented calculations for a disturbance of both semi-infinite width and finite width. In these later papers, he shows that the effects on the received signal depend on the distance of the disturbance from the receiver and transmitter. This same subject is treated in this paper, using a somewhat different approach.

\section{Application of Fresnel Diffraction Theory}

In this paper the approach is that used in textbooks on optics [e.g., Wood, 1934] in discussing diffraction by a thin wire or other opaque object. Here, however, the analogous diffracting object will be transparent rather than opaque, and will introduce a phase delay which differs from that which would be obtained in its absence.

In this application, it will be assumed that each elementary portion, $d s$, of the initially cylindrical wavefront emitted by the transmitter reradiates uniformly in all directions. Thus, the signal observed at the receiver is the sum of the signals reradiated by each element of the wavefront. The effect of the disturbance is to cause a phase change in that portion of the wavefront covered by the disturbance. The first step is to determine the phase of the signal reradiated by an element of the wavefront, $d s$, situated at a distance, $s$, from the line joining the transmitter to the receiver. The geometry of the situation is shown in figure 1, in which $T$ and $R$ are the transmitter and receiver, respectively. The phase disturbance is centered at $P$, and $P Q$ is a portion of the cylindrical wavefront from the transmitter. Thus, $T P$ and $T Q$ are equal to $a$. The distance $Q R$ is $b$, while $P R$ is $b+\delta$. $P U$ is a line perpendicular to $T R$ drawn through $P$.

The geometry of the figure shows that

$$
P R^{2}=U R^{2}+P U^{2}
$$

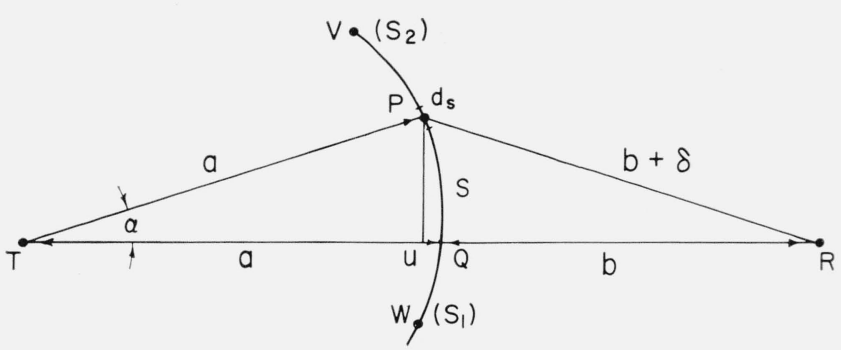

Figure 1. The geometry of the propagation of a wavefront VW from the transmitter $\mathrm{T}$ to receiver $\mathrm{R}$.

or

$$
(b+s)^{2}=(a+b-a \cos \alpha)^{2}+(a \sin \alpha)^{2} .
$$

Since

$$
\begin{aligned}
& \alpha \cong s / a, \\
& \delta=s^{2}(a+b) / 2 a b .
\end{aligned}
$$

Then the field at $R$, due to $d s$, is

$$
E=E_{0} \sin \left[\omega t-\frac{2 \pi}{\lambda}(a+b+\delta)\right] d s,
$$

where $\left|E_{0}\right|$ is a constant relating the observed field to the transmitted power, etc.

Thus, the total field due to the portion of the wavefront between $V$ and $W$, for which the distances, $s$, from $Q$ are $s_{2}$ and $s_{1}$, is given by

$$
E_{t}=E_{0} \int_{s_{1}}^{s_{2}} \sin \left[\omega t-\frac{2 \pi}{\lambda}(a+b+\delta)\right] d s
$$

which on expanding the sine term can be written in the form

$$
E_{t}=R E_{0} \sin \left[\omega t-\frac{2 \pi}{\lambda}(a+b)-\theta\right]
$$

where

$$
R^{2}=\left[\int_{s_{1}}^{s_{2}} \cos \frac{2 \pi \delta}{\lambda} d s\right]^{2}+\left[\int_{s_{1}}^{s_{2}} \sin \frac{2 \pi \delta}{\lambda} d s\right]^{2}
$$

and

$$
\theta=\tan ^{-1} \frac{\int_{s_{1}}^{s_{2}} \sin \frac{2 \pi \delta}{\lambda} d s}{\int_{s_{1}}^{s_{2}} \cos \frac{2 \pi \delta}{\lambda} d s} .
$$

The integrals are Fresnel's integrals and can be evaluated from tables, as discussed later.

It should be pointed out that, for several reasons, eq (2) is only approximate. First of all, the integral should contain a coefficient $1 \sqrt{b}$, which is dependent on $s$. Secondly, there should be an obliquity factor of the form $1 / 2(1+\cos$ TPR $)$ [Longhurst, 1957] to take account of the fact that the elements of the wavefront do not radiate equally in all directions. 
For the type of applications for which this work is intended, however, these details are unimportant since the angle $\alpha$ in figure 1 will be relatively small. One further objection to the present treatment might be that the phase of the received signal is not given correctly. This again is not important since it is only changes in the phase that are required. However, the correct phase could be obtained by permitting each element of the wavefront to reradiate with a phase lead of $\frac{\pi}{2}$ relative to the exciting signal. These refinements are included in Kirchoff's formulation of Huygen's principle [Longhurst, 1957].

Let us now consider an example in which the wavefront extends from $s_{0}$ to $s_{3}$. Between $s_{1}$ and $s_{2}$ the height of the ionosphere is lowered so that in this region the phase of the signal is advanced by an amount, $\varphi$ [Wait, 1961], but between $s_{0}$ and $s_{1}$, and $s_{2}$ and $s_{3}$, it is unchanged. For brevity let

and let

$$
\begin{aligned}
& E_{0} \sin \left[\omega t-\frac{2 \pi}{\lambda}(a+b)\right]=A, \\
& E_{0} \cos \left[\omega t-\frac{2 \pi}{\lambda}(a+b)\right]=B
\end{aligned}
$$

$$
s=\sqrt{\frac{a b \lambda}{2(a+b)}} v .
$$

Then using eq (1) and the definitions

$$
\begin{aligned}
& \int_{0}^{v_{1}} \cos \left(\frac{\pi v^{2}}{2}\right) d v=C\left(v_{1}\right), \\
& \int_{0}^{v_{1}} \sin \left(\frac{\pi v^{2}}{2}\right) d v=S\left(v_{1}\right),
\end{aligned}
$$

for the two Fresnel integrals, eq (2) can be written in the form,

$$
\begin{aligned}
E_{t}=A\left\{C\left(v_{1}\right)-C\left(v_{0}\right)+\cos \varphi\left[C\left(v_{2}\right)-C\left(v_{1}\right)\right]\right. & \\
& \left.+\sin \varphi\left[S\left(v_{2}\right)-S\left(v_{1}\right)\right]+C\left(v_{3}\right)-C\left(v_{2}\right)\right\} \\
-B\left\{S\left(v_{1}\right)-\right. & S\left(v_{0}\right)+\cos \varphi\left[S\left(v_{2}\right)-S\left(v_{1}\right)\right] \\
& \left.+\sin \varphi\left[C\left(v_{2}\right)-C\left(v_{1}\right)\right]+S\left(v_{3}\right)-S\left(v_{2}\right)\right\}
\end{aligned}
$$

or

Thus,

$$
E_{t} \equiv A D-B F
$$

$$
E_{t}=\sqrt{D^{2}+F^{2}} \cdot E_{0} \sin \left[\omega t-\frac{2 \pi}{\lambda}(a+b)-\theta\right]
$$

where

$$
\theta=\tan ^{-1} F / D \text {. }
$$

The change of phase $\Delta \theta$ in the received signal is thus

$$
\Delta \theta=\theta-\frac{\pi}{4} \text { since } \theta=\frac{\pi}{4} \text { when } \varphi=0 .
$$

We can now consider some special cases. First of all, let $\varphi=0$, which represents the undisturbed case. Then,

$$
\begin{aligned}
& D=\left[-C\left(v_{0}\right)+C\left(v_{3}\right)\right], \\
& F=\left[-S\left(v_{0}\right)+S\left(v_{3}\right)\right] .
\end{aligned}
$$

To include the whole wavefront, let $s_{0}$ and $s_{3}$ become very large. Then,

$$
v_{0} \rightarrow-\infty \text { and } v_{3} \rightarrow+\infty \text {. }
$$

Thus, $D=1, F=1$, and $\theta=\frac{\pi}{4}$. Since

$$
C( \pm \infty)= \pm \frac{1}{2}, S( \pm \infty)= \pm \frac{1}{2} .
$$

This shows that the total field produced by the unperturbed wavefront lags the field due to the portion of the wavefront at $Q$ by $\frac{\pi}{4}$ and has a value $\sqrt{2} E_{0}$.

Secondly, consider the case in which $v_{0}=v_{1}=-\infty$ and $v_{2}=v_{3}=+\infty$. This represents the case in which the whole wavefront is advanced in phase by an amount $\varphi$.

Then,

$$
\begin{aligned}
& D=\cos \varphi+\sin \varphi, \\
& F=\cos \varphi-\sin \varphi .
\end{aligned}
$$

Thus,

$$
\begin{aligned}
R & =\sqrt{2}, \\
\theta & =\frac{\pi}{4}-\varphi, \text { and } \Delta \theta=-\varphi,
\end{aligned}
$$

which shows that the phase of the received signal was advanced by an amount $\varphi$, as might be expected.

Thirdly, we consider the more general case in which

Then,

$$
v_{0}=-\infty, v_{3}=+\infty .
$$

$$
\begin{aligned}
D=\left\{1-\left[C\left(v_{2}\right)-C\left(v_{1}\right)\right](1\right. & -\cos \varphi) \\
+ & {\left.\left[S\left(v_{2}\right)-S\left(v_{1}\right)\right] \sin \varphi\right\} }
\end{aligned}
$$

and

$F=\left\{1-\left[S\left(v_{2}\right)-S\left(v_{1}\right)\right](1-\cos \varphi)\right.$

$$
\left.-\left[C\left(v_{2}\right)-C\left(v_{1}\right)\right] \sin \varphi\right\} .
$$

These expressions for $D$ and $F$, and consequently for $R$ and $\theta$, can be evaluated numerically with the aid of a table of Fresnel integrals, or graphically by means of Cornu's spiral, as shown in figure 2. Consider a disturbance which extends from $s_{1}$ to $s_{2}$, where, as in figure $1, s_{1}$ and $s_{2}$ are the distances from the edges of the disturbance to the line $T R$. In this case, however, it is assumed that the entire disturbance is on one side, only, of the line TR. By means of (6), the equivalent values $v_{1}$ and $v_{2}$, of $s_{1}$ and $s_{2}$ are obtained and marked on the Cornu spiral in figure 2 . The contribution to the received signal from $v=-\infty$ 


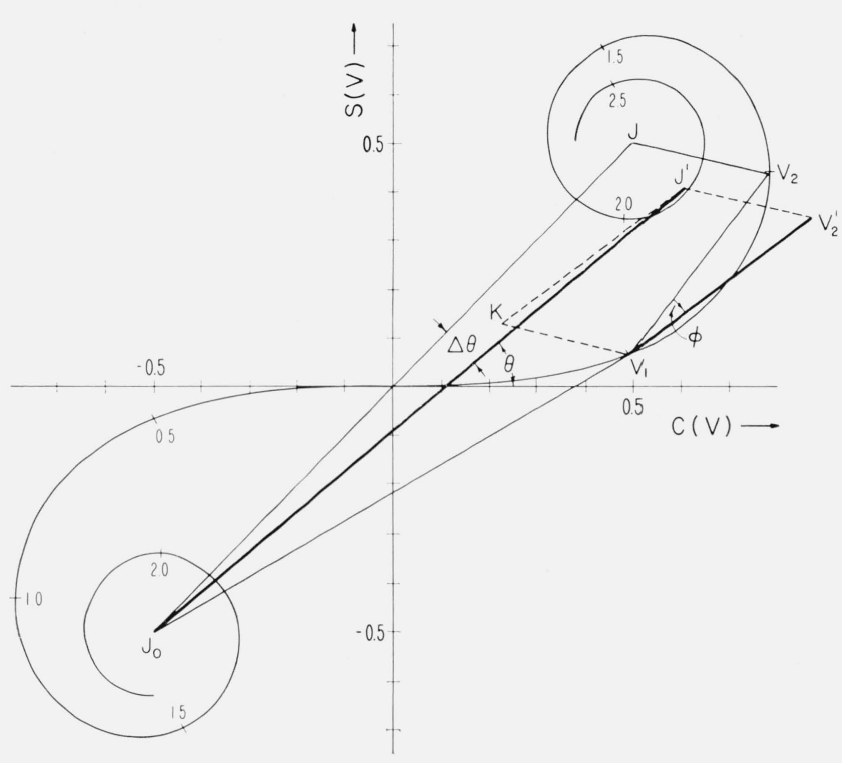

Figure 2. Cornu spiral.

The received signal is represented in phase and amplitude by $J_{0} J$ when the wavefront is undisturbed. The effect of a phase advance $\varphi$ on the wavefront between $v_{1}$ and $v_{2}$ is to rotate $v_{2}$ to $v_{2}^{\prime}$. The received signal is now given by $J_{0} J$ which is the vector sum of the unchanged components $J_{0} v_{1}+v_{2} J=J_{0} k$, and the altered component $v_{1} v_{2}^{\prime}$.

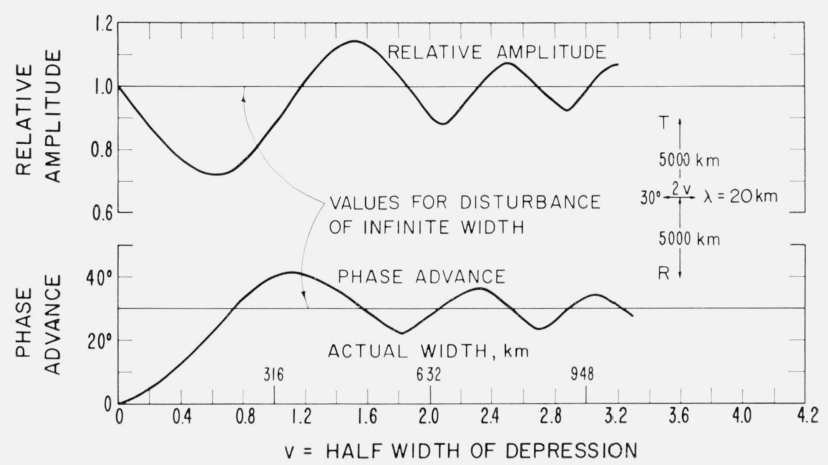

Figure 3. The effects of a uniform disturbance (for which $\left.\varphi=30^{\circ}\right)$ centered on the path.

The actual width, $2 s$, is obtained from eq (7); the numerical values shown refer only to the specific case illustrated on the right.

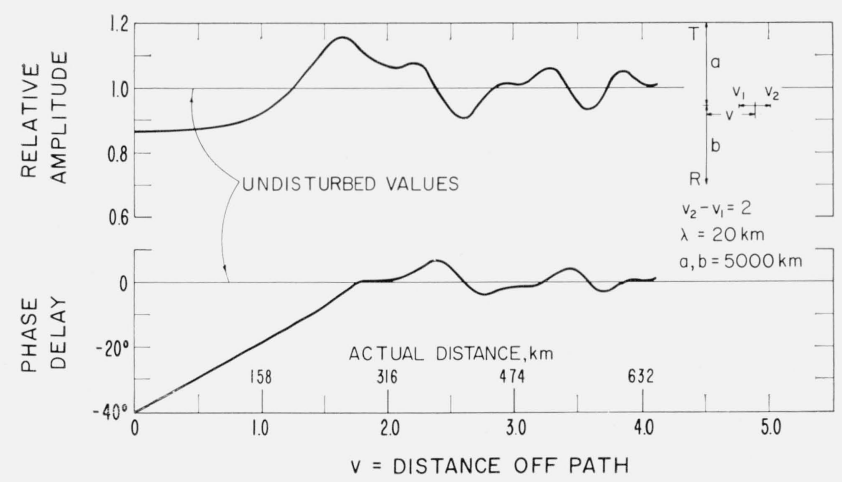

Figure 4. The effects of a uniform disturbance (for which $\varphi=30^{\circ}$ ) on the received signal as it moves across the path.

The disturbance is of fixed width, $v_{2}-v_{1}=2$ which in the case illustrated is 316 $\mathrm{km}$. The actual distances given refer only to the specific case illustrated on the right. to $v=v_{1}$ is given by the chord $J_{0} v_{1}$. The contribution from the wavefront between $v_{1}$ and $v_{2}$ is given by $v_{1} v_{2}$. The effect of the phase advance, $\varphi$, along the region of the wavefront between $v_{1}$ and $v_{2}$ is obtained by rotating $v_{1} v_{2}$ by the amount, $\varphi$, to the position, $v_{1} v_{2}^{\prime}$. (This follows from (8).) Since the remainder of the wavefront between $v=v_{2}$ and $+\infty$ is given by $v_{2} J$, this is added vectorially to $J_{0} v_{2}^{\prime}$, and $J$ is moved to $J^{\prime}$. Then the final resultant is given by $J_{0} J^{\prime}$, both in amplitude and phase. In the absence of the phase change between $v_{1}$ and $v_{2}$, the resultant signal is given by $J_{0} J$. Thus, the effect of the phase perturbation on amplitude is $\left|J_{0} J^{\prime}\right| /\left|J_{0} J\right|$, and the resultant phase change is the angle $\Delta \theta$ between $J_{0} J$ and $J_{0} J^{\prime}$.

The results of some calculations made in this way are shown in figures 3 and 4 . The first of these refers to a phase advance which is symmetrically situated about the center of the path. The width of the wavefront $\left(v=v_{1}=-v_{2}\right)$ suffering the phase advance is the variable. The calculations refer to the case where $a=b=5000 \mathrm{~km}, \lambda=20 \mathrm{~km}$ and $\varphi=30^{\circ}$. It will be seen that it is not until the width of the disturbance $\left(v_{2}-v_{1}\right)$ approaches about 1.5 that the full effect is observed at the receiver. As the width increases further, the phase and amplitude of the signal at the receiver oscillate about the value which it would have if the phase advance extended from $v=-\infty$ to $+\infty$.

Figure 4 shows another example. In this case, a phase advance $\left(\varphi=30^{\circ}\right)$ of fixed width, $v_{2}-v_{1}=2$, is considered, but its center is moved across the path. The figure shows that as the center of the disturbance moves away from the path, the initial perturbations decrease from the value given in figure 3. At sufficient distances from the path, the phase and amplitude merely oscillate about the values which would be observed in the absence of the ionospheric perturbation. The somewhat peculiar shape of the oscillation is due to the fact that the Fresnel integrals are not periodic for small values of $v$.

Figures 3 and 4 have been calculated for the case in which the disturbance is equidistant from the ends of the path so that $a=b$. Other cases in which $a \neq b$ are easily considered by merely decreasing the value of $s$ equivalent to a given value of $v$, in accordance with (6). Thus the width of a disturbed region of the wavefront necessary to produce a given effect is reduced as the disturbance approaches either the transmitter or receiver. Furthermore, for off-path disturbances, the distance from the path at which a particular effect is produced is reduced as the disturbance moves towards either end of the path. Equation (6) also shows that a decrease in wavelength has the same effect as moving the disturbance towards one end of the path.

\subsection{Effects of Circular Depressions}

In the preceding sections, the profile of the disturbance in height of the ionosphere has been specified by the phase change, $\varphi$, that would be suffered by a ray of infinitesimal width passing through it [Wait, 1961]. The effect of varying the width of 
the disturbed region has then been discussed as though the width $\left(s_{2}-s_{1}\right)$ or $\left(v_{2}-v_{1}\right)$, and $\varphi$ were independent.

As a first attempt to investigate a more realistic case in which $\left(s_{2}-s_{1}\right)$ and $\varphi$ are not independent, consider a depression which is a square with a side length of $d$ when viewed in plan. Then, since $\varphi$ increases as the length of path through the disturbance increases, $\varphi$ tends to be proportional to $d$. However, as the observed phase change, $\theta$, also tends to increase with $d$, the overall effect, when $d$ is small, is for $\theta$ to increase approximately as $d^{2}$. If the depth of the depression also increases with $d$, the dependence is even stronger.

In the case of a circular disturbance, there is a further complication since the phase change suffered by a ray now depends on the distance from the center at which it passes through the disturbance. It is a maximum for the ray passing through the center, but zero for the tangent ray. Furthermore, in most cases, the depth of the depression will be a maximum at the center and will smoothly decrease until it is zero at the edges. Thus the dependence of phase shift on distance from the center may become marked, and under these circumstances, it might be regarded that the "effective" diameter is much less than the actual diameter. This effect will be illustrated in the next paragraph.

Wait [1961] has given expressions which enable the phase change produced by an infinitely wide depression having a vertical profile of the form

$$
\begin{aligned}
& h=h_{0} \text { for } x>x_{0} \\
& h=h_{0}-\Delta h \cos \left(\frac{\pi}{2} \frac{x}{x_{0}}\right), \text { for } x<x_{0}
\end{aligned}
$$

to be obtained. In this, $h_{0}$ is the height of the undisturbed ionosphere, $\Delta h$ is the maximum depression, $x$ is the distance from the center of the depression, and $2 x_{0}$ is the width of the depression, both measured in the direction of propagation. Wait has calculated that such a depression, for which $x_{0}=400 \mathrm{~km}$, $\Delta h=20 \mathrm{~km}, h_{0}=80 \mathrm{~km}$, and $\lambda=20 \mathrm{~km}$, would advance the phase of an infinitesimally narrow ray by $+26^{\circ}$. Consider now a circular depression whose central cross section is of the same cosinusoidal form as that just discussed, i.e., of diameter $2 x_{0}$ and depth $\Delta h$. A chord drawn horizontally through this depression at a distance $x$ from the center will now have a height profile given by

$$
h^{\prime}=h_{0}-\Delta h^{\prime} \cos \left(\frac{\pi x^{\prime}}{4 \sqrt{x_{0}^{2}-x^{2}}}\right)
$$

where $x^{\prime}$ is the distance from the center of the chord, $2 \sqrt{x_{0}^{2}-x^{2}}$ is the length of the chord and $\Delta h^{\prime}=\Delta h \cos$ $\left(\frac{\pi}{2} \cdot \frac{x}{x_{0}}\right)$.

To a first approximation $h^{\prime}$ is a cosinusoidal function of $x^{\prime}$. Thus the formulas given by Wait [1961] can be used for obtaining the phase delay through the disturbance, after calculating the appropriate values of $\Delta h^{\prime}$ and $\sqrt{x_{0}^{2}-x^{2}}$, for each value of $x$. The results of calculating in this way the phase advance for a narrow ray passing through the circular disturbance discussed above are given in table 1.

TABle 1. Phase change through an $800 \mathrm{~km}$ diameter, $20 \mathrm{~km}$ deep cosinusoidal depression at varying distances from its center

\begin{tabular}{l|r|r|r|r|r|r}
\hline \hline $\begin{array}{l}\text { Distance from center } \\
\text { Phase advance }\end{array}$ & 400 & 320 & 240 & 160 & 80 & $0 \mathrm{~km}$ \\
\hline
\end{tabular}

By averaging these phase advances over the width of this disturbance, it is found that the same average phase advance would be produced by a depression only $320 \mathrm{~km}$ wide, if its cross section along the direction of propagation is everywhere the same as the cross section through the center of the circular depression.

The actual effects on the received signal produced by a nonuniform disturbance, such as the one discussed above, can be determined by using, instead of (10), the following expressions.

$$
\begin{array}{r}
D=\left\{1+C\left(v_{1}\right)-C\left(v_{m}\right)+\sum_{n=2}^{m} \cos \phi_{n}\left[C\left(v_{n+1}\right)-C\left(v_{n}\right)\right]\right. \\
\left.\left.+\sum_{n=2}^{m} \sin \phi_{n}{ }^{\prime} S\left(v_{n+1}\right)-S\left(v_{n}\right)\right]\right\} \\
F=\left\{1+S\left(v_{1}\right)-S\left(v_{m}\right)+\sum_{n=2}^{m} \cos \phi_{n}\left[S\left(v_{n+1}\right)-S\left(v_{n}\right)\right]\right. \\
\left.-\sum_{n=2}^{m} \sin \phi_{n}\left[C\left(v_{n+1}\right)-C\left(v_{n}\right)\right]\right\} \cdot(11)
\end{array}
$$

In these expressions, the disturbed portion of the wavefront is divided into $m$ small portions, and $\phi_{n}$ is the phase delay produced by the section between $v_{n}$ and $v_{n+1}$.

\subsection{Effects Due to the Curvature of the Earth}

In calculating the phase anomaly caused by a cosinusoidal depression in the ionosphere, Wait [1961] included the effects of the earth's curvature on phase velocity. In the present case, however, there is a further geometrical effect which follows from the fact that in figure 1 the triangle $T P R$ is spherical. If the side lengths are expressed in angular measure and denoted by $\hat{a}, \hat{b}$, etc., then it is easily shown, using spherical trigonometry, that (1) now becomes

$$
\hat{\delta}=\hat{s}^{2} \frac{(\tan \hat{a}+\tan \hat{b})}{2 \tan \hat{a} \cdot \tan \hat{b}} .
$$


On substituting

$$
\hat{s}=\sqrt{\frac{\tan \hat{a} \cdot \tan \hat{b} \hat{\lambda}}{2(\tan \hat{a}+\tan \hat{b})}} \cdot v,
$$

we again find that $\frac{2 \pi \hat{\delta}}{\hat{\lambda}}=\frac{2 \pi \delta}{\lambda}=\frac{\pi v^{2}}{2}$ and the remainder

of the formulas are unchanged. The consequence of this is that $s=\hat{s} \cdot r$ (where $r$ is the radius of the earth) must be increased to obtain a particular value of $v$. Indeed, as $a$ and $b$ both approach $10,000 \mathrm{~km}\left(\frac{a}{r}=\frac{\pi}{2}\right)$, the value of $s$ required for a finite $v$ approaches infinity. Under these conditions, the size of the disturbance necessary to produce a given effect on the received signal becomes very large. It is worth noting, however, that $a$ or $b$ separately may approach $10,000 \mathrm{~km}$ since (13) reduces to

$$
s=\sqrt{\frac{\hat{\lambda} \tan \hat{b}}{2(1+\tan \hat{a} / \tan \hat{b})}} \cdot v
$$

or

$$
s=r \sqrt{\frac{\hat{\lambda} \tan \hat{b}}{2}} \cdot v
$$

when $\hat{a} \rightarrow \frac{\pi}{2}$, and similarly for $\hat{b} \rightarrow \frac{\pi}{2}$. Thus when either the receiver or transmitter is about $10,000 \mathrm{~km}$ from the ionospheric depressions, finite results will be observed.

For paths of moderate lengths, the effect of the curvature of the earth is quite small. For example, consider the case in which $a=b=5000 \mathrm{~km}, \lambda=20$ $\mathrm{km}$, and $r=6400 \mathrm{~km}$. Then for a flat earth (6) shows that $s=158 \mathrm{v} \mathrm{km}$, while (13), for the curved earth, gives $s=180 v \mathrm{~km}$. The differences become greater, of course, as $a$ and $b$ increase.

\section{Discussion}

The main purpose of this paper is to enable estimates to be made of the effects of a particular disturbance as its position relative to the radio path is changed. Such estimates are best given by comparison with the effects which would be observed in the hypothetical case of the received signal consisting of an infinitely narrow ray passing through the center of the disturbance. The total signal at the receiver is the vector sum of two portions: that portion which is not altered by the disturbance, and that portion which is altered. In figure 2 , the vector sum $\overline{J_{0} K}$ of $\overline{J_{0} v_{1}}$ and $\overline{v_{2} J}$ comprises the undisturbed portion of the field, while $\overline{v_{1} v_{2}}$ is the portion which is modified by rotation of $v_{2}$ to $v_{2}^{\prime}$ through the angle $\varphi$. As a general principle, then, from an examination of the Cornu spiral in figure 2, it would seem that for the phase of the vector sum of the whole wave to differ from that due to the wholly undisturbed wave by an amount approaching $\varphi$, the length $\overline{v_{1} v_{2}}=\overline{K J^{\prime}}$ must be greater than $\overline{J_{0} v_{1}}+\overline{v_{2} J}=\overline{J_{0} K}$. This means, in essence, that a disturbance centered on the path must extend from $v_{1}=0.7$ to $v_{2}>+0.7$, approximately, while the nearest edge of an off-path disturbance must be close enough to the path that $v_{1}$ be less than unity. These figures are, of course, rough approximations and are given only as a guide; in the case of off-path disturbances, in particular, estimates should be made from the Cornu spiral for each special case.

It should be noted also that there is, in general, no simple proportionality between the phase change $\varphi$ of the disturbance, and the phase change $\Delta \theta$ of the total signal. Examination of figure 2 shows, in fact, that the maximum possible phase change, $\Delta \theta^{\prime}$, in the received signal is approximately $\Delta \theta^{\prime}=$ $\tan ^{-1} \frac{\overline{v_{1} v_{2}}}{\overline{J_{0} K}}$ and occurs when $\overline{v_{1} v_{2}^{\prime}}$ is at right angles to $\overline{J_{0} K}$

It is perhaps also worthy of mention that if the ionospheric disturbance introduces attenuation as well as a change of phase, this can be readily taken into account. Graphically, as in figure 2, for example, the attenuation can be included by simply reducing the length of the disturbed vector $v_{1} v_{2}^{\prime}$ at the same time as it is rotated.

\section{Conclusions}

The effects of localized ionospheric depressions on the phase and amplitude of a VLF signal have been discussed in terms of elementary Fresnel diffraction theory. This has been made possible by two main simplifications: (1) by assuming that only one waveguide mode is present and that the only effect of the depression in the ionosphere is to locally alter the phase velocity of the waveguide mode, and (2) that the ionospheric depression is sufficiently small that the change in phase velocity caused by it can be represented as a "lumped" phase change in the wavefront where it passes through the disturbance.

It has been shown that:

(1) Even when the center of the disturbance lies along the path, it must be of finite dimensions before the phase and amplitude of the received signal are affected by it. A small circular disturbance produces an effect which initially increases about as the square of its diameter.

(2) When the center of the disturbance is not on the path, its near edge must pass within a certain distance of the path to produce significant effects. Otherwise, as the distance of the disturbance from the path increases, both the amplitude and phase oscillate about their undisturbed values, and phase retardations are as likely to be observed as phase advances. Indeed, when the center of the disturbance is at certain distances away from the path, the phase of the received signal may show no change at all. Under these circumstances, however, the amplitude of the received signal will be changed. 
(3) A disturbance produces the smallest effects when it is equidistant from the ends of the path. As it is moved nearer one end or the other, its effects on the received signal increase. An off-path disturbance must, however, move nearer the path as it moves towards either end of the path if it is to maintain the same effect. It is also easily shown that the effects of a given on-path disturbance are greater at higher frequencies, but again, off-path disturbances must be closer to the path than at lower frequencies to produce the same effect.

(4) A geometrical effect due to the curvature of of the earth causes the critical dimensions (diameter and distance off-path) to be increased above those which would be calculated on a flat earth basis to produce a change of given magnitude. In particular, a disturbance at the center of the path, which is about $20,000 \mathrm{~km}$ long, will only produce very small effects.

Most of these results seem to be in agreement with those obtained independently by Wait [1964 $\mathrm{a}, \mathrm{b}]$.
This work was done at the suggestion of A. G. Jean and was supported by the Advanced Research Projects Agency, Washington, D.C., under Order 183.

\section{References}

Longhurst, R. S. (1957), Geometrical and physical opties, Longmans, Green and Coy, New York, 190.

Wait, J. R. (Oct. 1961), Expected influence of a localized change of ionosphere height on VLF propagation, J. Geophys. Res. 66, 3119.

Wait, J. R. (Jan. 1964a), Calculated diffraction effects at VLF from a localized ionospheric depression, NBS Tech. Note No. 208.

Wait, J. R. (Feb. 1964b), On phase changes in VLF propagation induced by an ionospheric depression of finite extent, J. Geophys. Res., 69, 441.

Wood, R. W. (1934), Physical optics, 3d edition, p. 231, MacMillan, N.Y.

(Page 68D6-368) 